\title{
Determinação do caseinomacropeptídeo em leite UAT por espectroscopia no infravermelho próximo e regressão por mínimos quadrados parciais
}

\author{
[Determination of caseinomacropeptide in uht milk using near infrared spectroscopy \\ and pls regression] \\ R.R. Oliveira ${ }^{1}$, M.I.A. Souz ${ }^{2}$, C.H. Silva ${ }^{3}$, C.S. Prado ${ }^{3}$, C.S.M. Rezende ${ }^{3}$, M.E. Lage ${ }^{3}$ \\ ${ }^{1}$ Centro Universitário de Anápolis - Anápolis, GO \\ ${ }^{2}$ Aluna de pós-graduação - Universidade Federal de Goiás - Goiânia, GO \\ ${ }^{3}$ Universidade Federal de Goiás - Goiânia, GO \\ RESUMO
}

\begin{abstract}
O presente trabalho foi realizado com o objetivo de desenvolver um método para a determinação de CMP em leite UAT por meio da aplicação da espectroscopia de infravermelho próximo. Leites UAT de oito marcas diferentes foram utilizados para a construção dos modelos de calibração. Os resultados demonstram que, para o desenvolvimento de um modelo de calibração adequado para a determinação de CMP em leite UAT, deve-se utilizar, juntamente com o método de regressão PLS, o método de seleção de espectros máxima distância e os pré-tratamentos $2^{\mathrm{a}}$ derivada e variável normal padronizada. Além disso, pôde-se determinar que as regiões do infravermelho próximo mais correlacionadas com os movimentos vibracionais dos aminoácidos presentes no CMP foram: 1100-1310; 1400-1430; 1490-1550; 1640-1680; 1780-1970; 2020-2100 e 2310-2350nm. Conclui-se que a espectroscopia de infravermelho próximo pode ser uma alternativa para a determinação de CMP em leite UAT, desde que haja um conjunto de calibração com amostras representativas da população a ser predita no futuro.
\end{abstract}

Palavras-chave: calibração multivariada, fraude, NIRS, soro de queijo

\section{ABSTRACT}

This work's objective was to develop an UHT milk caseinomacropeptide determination method trough NIR spectroscopy application. Eight UHT milk trademarks are used to produce a mathematical calibration model. The results of NIR analysis suggested that to produce a suitable calibration model, partial least-square regression (PLSR) must be used, with maximum distance in wavelenght space to select spectra, pre - treatment with 2nd derivative and standard normal variant (SNV). Also, suitable near-infrared regions more correlated with CPM aminoacids vibrational movements: 1100-1310; 14001430; 1490-1550; 1640-1680; 1780-1970; 2020-2100; and 2310-2350nm. Therefore, NIR spectroscopy can be an alternative to caseinomacropeptide determination of UHT milk, since there was a representative calibration set with a large enough and representative sample of entire population to be predicted in the future.

Keywords: fraud, multivariate calibration, NIRS, whey

\section{INTRODUÇÃO}

A adição do soro de queijo ao leite fluido é uma das fraudes em alimentos mais praticadas no Brasil (Valente et al., 2014). Esse tipo de adição ao leite pode ser detectado e quantificado pela determinação do caseinomacropeptídeo (CMP), em que se tolera um índice de CMP de até
30mg/L para o leite a ser destinado ao abastecimento direto (Brasil, 2006a).

Entretanto, a determinação do CMP em leite requer a utilização de análises demoradas, caras e difíceis de serem executadas (Brasil, 2006b; Brasil, 2014). Como a indústria necessita de respostas rápidas, foram objeto de estudos nos últimos anos técnicas que exijam menor

Recebido em 1 de fevereiro de 2017

Aceito em 25 de agosto de 2017

E-mail: rapha.vet@gmail.com 
manipulação, preparo e tempo de análise reduzidos, além de bom grau de precisão. Nesse sentido, a técnica de espectroscopia de infravermelho próximo (NIRS) apresenta-se como uma alternativa promissora (Zhang et al., 2014; Botelho et al., 2015).

O espectro obtido a partir do NIRS é o resultado da reflexão da luz por moléculas orgânicas. Todas as bandas de absorção/reflexão são resultantes de vibrações ou combinações de vibrações (Skoog et al., 2002). Como os alimentos apresentam grande número de ligações moleculares que caracterizam diversos compostos orgânicos, mas que absorvem luz de um mesmo comprimento de onda, gerando espectros com combinações e forte sobreposição das bandas de vibração e harmônicas, os espectros NIRS não são facilmente interpretáveis, o que inviabilizou a técnica até a década de 80 (Van kempen e Jackson, 1996).

Com a popularização dos microcomputadores e o surgimento de uma nova área na química, a quimiometria, o infravermelho próximo voltou a ser objeto de estudos em química analítica (Natalino, 2006), já que a quimiometria utiliza métodos matemáticos, estatísticos e de lógica formal, a fim de selecionar os melhores procedimentos experimentais para a obtenção de informações químicas de natureza multivariada (Varmuza e Filzmoser, 2009).

A espectroscopia no infravermelho já foi aplicada em leite fluido para as determinações de proteína, caseína total, gordura, lactose, umidade e para a realização da classificação do leite (Klandar et al., 2007; Cipolat-Gotet et al., 2012; Lyndgaard et al., 2012), além de detectar adulterações por adições de proteína vegetal e amido (Li e Ding, 2010).

No entanto, não foram encontrados estudos utilizando o método NIRS para a determinação de fraudes por adição de soro ao leite fluido. Dessa forma, objetivou-se, com o presente estudo, desenvolver um método com boa capacidade preditiva para a determinação do caseinomacropeptídeo em leite UAT, por meio da espectroscopia no infravermelho próximo e regressão por mínimos quadrados parciais.

\section{MATERIAL E MÉTODOS}

O presente trabalho foi desenvolvido no Centro de Pesquisa em Alimentos da Escola de Veterinária e Zootecnia da Universidade Federal de Goiás (EVZ/UFG). Foram utilizados no experimento leites UAT de oito marcas diferentes, todos oriundos de laticínios sob Inspeção Federal (SIF), sendo sete localizados no estado de Goiás e um em Minas Gerais. As amostras das marcas de leites UAT foram adquiridas e mantidas em temperatura ambiente e, no momento das análises, apresentavam período de fabricação inferior a um mês.

Todas as amostras foram analisadas por cromatografia líquida de alta eficiência (CLAE), em triplicata, para que fosse determinado $o$ índice de CMP em cada leite UAT.

Para a determinação em $\mathrm{mg} / \mathrm{L}$ do teor de CMP por CLAE em leite UAT, foi feita uma curva de calibração. Para o seu preparo, leite genuíno, colhido na fazenda da EVZ/UFG, foi adulterado com soro de queijo, nas proporções de $5 \%, 10 \%$, $15 \%$ e $20 \%$, conforme recomendado pela metodologia oficial (Brasil, 2006b). O soro de queijo utilizado na elaboração da curva padrão foi obtido após a adição do coalho em pó e subsequente coagulação da caseína em leite genuíno, colhido na fazenda da EVZ/UFG momentos antes das análises. A curva de calibração construída apresentou um valor de $\mathrm{R}^{2}$ de 0,9947 .

A determinação cromatográfica foi realizada em cromatógrafo GILSON 118, utilizando-se detector UV/VIS operando em vazão de $1 \mathrm{~mL} / \mathrm{min}$, e a detecção na faixa do UV em comprimento de onda de $205 \mathrm{~nm}$, com a linha de base já devidamente estabilizada. A corrida cromatográfica foi realizada em aparelho de cromatografia líquida de alta eficiência, com sistema isocrático (GILSON 306), com injetor automático ASTED - XL da marca GILSON e looping de $20 \mu \mathrm{L}$ e coluna Zorbax GF-250 BIO Séries da Agilent de $9 \mathrm{~mm}$ de diâmetro interno por $250 \mathrm{~mm}$ de comprimento. A fase estacionária da coluna era composta por partículas esféricas de sílica, modificadas na superfície por zircônio estabilizado, enquanto a fase ligada era composta por monocamada molecular hidrofílica, com diâmetro de poro de $150 \mathrm{~A}$. A solução da fase móvel usada foi um tampão fosfato com $\mathrm{pH} 6$ $\left(1,74 \mathrm{~g}\right.$ de fosfato de potássio dibásico $\left(\mathrm{K}_{2} \mathrm{HPO}_{4}\right)$ 
p.a., $12,37 \mathrm{~g}$ de fosfato de potássio monobásico $\left(\mathrm{KH}_{2} \mathrm{PO}_{4}\right)$ p.a. e $21,41 \mathrm{~g}$ de sulfato de sódio $\left(\mathrm{Na}_{2} \mathrm{SO}_{4}\right)$ dissolvido em $1000 \mathrm{~mL}$ de água milli-Q ultrapurificada.

Esse procedimento foi denominado de tratamento 0 , pois não houve adição de soro, as amostras foram analisadas como obtidas do mercado. Os resultados encontrados variaram de 33,64 a $398,78 \mathrm{mg} / \mathrm{L}$.

Além do tratamento 0, para cada marca foram obtidos outros sete tratamentos, com base nas adições de soro de queijo reconstituído ao leite em $1 \%, 2 \%, 3 \%, 4 \%, 6 \%, 8 \%$ e $10 \%$. O soro utilizado foi obtido por meio da reconstituição de soro doce em pó, de uma marca comercial. Como o soro doce contém entre 6 e $7 \%$ de sólidos totais (Carvalho et al., 2007), para a preparação do soro foram utilizados $70 \mathrm{~g}$ de soro em pó para cada litro de água destilada.

Para melhor compreensão, os tratamentos foram denominados conforme o percentual de adição do soro de queijo ao leite, ou seja, de tratamentos
$1,2,3,4,6,8$ e 10, respectivamente, totalizando, dessa forma, oito tratamentos para cada marca utilizada e 64 tratamentos no total.

O soro reconstituído foi analisado por CLAE e apresentou uma concentração média de $2.540 \mathrm{mg}$ por litro. A partir desse valor, foram realizados cálculos para se determinar a quantidade de CMP presente em $1 \%, 2 \%, 3 \%, 4 \%, 6 \%, 8 \%$ e $10 \%$ do soro reconstituído, sendo, respectivamente: 25,4 ; 50,8; 76,2; 101,6;152,4; 203,2; 254mg/L. Após as adições desses valores aos resultados encontrados para os tratamentos zero de cada marca, foram obtidos valores de CMP entre 33,64 e $652,78 \mathrm{mg} / \mathrm{L}$, para os 64 tratamentos que formaram o conjunto de calibração. A distribuição das concentrações de CMP das amostras do conjunto de calibração pode ser visualizada na Fig. 1.

As leituras das amostras por NIRS foram realizadas em um espectrofotômetro da Perstorp Analytical Company, modelo 6500, equipado com detector de reflectância, na faixa de 1100 a $2500 \mathrm{~nm}$, em intervalos de $2 \mathrm{~nm}$.

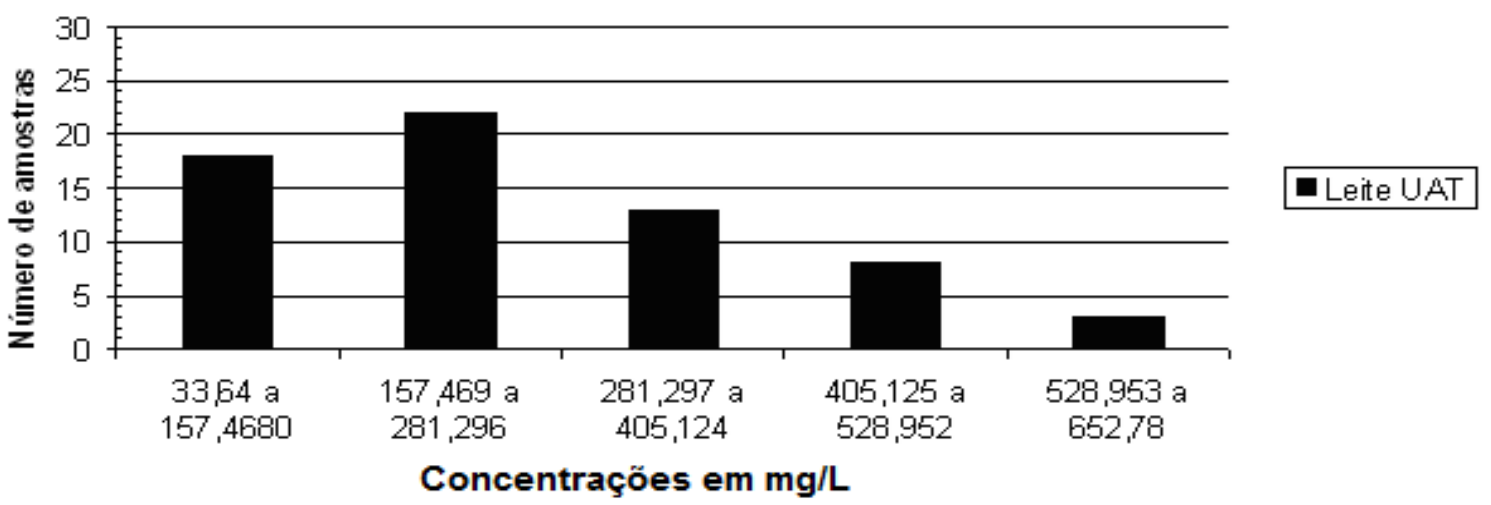

Figura 1. Distribuição de frequência das concentrações de CMP dos 64 tratamentos do conjunto de calibração.

O equipamento utilizado no presente trabalho só dispunha de células para análises de amostras sólidas. Para a utilização de amostras líquidas, foi desenvolvida uma célula em uma torneadora, em material de alumínio, com as mesmas dimensões de uma célula para sólidos, pintado com uma tinta preta fosca, para que, nas análises por NIRS, houvesse reflexões associadas apenas às amostras de leite UAT. A célula foi formada por um suporte e por uma tampa contendo o vidro de quartzo. $\mathrm{O}$ vidro de quartzo foi fixado à tampa com cola de silicone. Foram colocadas borrachas entre o suporte e a tampa, para proteger o vidro e permitir a vedação da peça, por meio do ajuste suporte/tampa por quatro parafusos localizados nas extremidades da célula projetada. Foi feita uma abertura na parte superior da peça, para facilitar o uso e o descarte das amostras de leites UAT. Na célula projetada, foram necessários apenas $45 \mathrm{~mL}$ de cada amostra.

Cada espectro foi obtido por NIRS em apenas 23 segundos. Para eliminar ruídos aleatórios, como ruídos da rede elétrica, as medidas no aparelho 
foram realizadas 32 vezes para cada amostra, sendo obtido o espectro médio. A fim de garantir que variações extremas de temperatura não interferissem na coleta dos espectros, o equipamento foi mantido em uma sala com temperatura controlada de, aproximadamente, $25^{\circ} \mathrm{C}$.

Em cada um dos 64 tratamentos, foram obtidos espectros em três replicatas, obtendo-se 192 espectros no total. Os espectros armazenados foram posteriormente submetidos aos tratamentos estatísticos disponíveis no software Vision $^{\circledR}$, em que foram desenvolvidos vários modelos para determinar o método de seleção de espectros, o(s) pré-tratamento(s) matemático(s) e as regiões do infravermelho próximo que deveriam ser utilizados para a obtenção do modelo de calibração mais adequado para a determinação de CMP em leite UAT.

$\mathrm{Na}$ seleção desses parâmetros, foram considerados, como critério inicial, os modelos que apresentaram maiores coeficientes de determinação $\left(\mathrm{R}^{2}\right)$. Nos casos em que os coeficientes de determinação mostraram-se próximos, utilizou-se o menor valor de PRESS e o menor número de fatores.

Inicialmente, os testes foram realizados para se definir o método de seleção de espectros mais adequado para a obtenção de um bom modelo de calibração. Nessa etapa, foram obtidos dois modelos, um com cada método de seleção de espectros, os quais podem ser observados no Quadro 1. Para o desenvolvimento desses modelos, além de um dos métodos de seleção, foram utilizados o método de regressão PLS e a informação contida na faixa de 1100 a 2500nm.

Quadro 1. Métodos de seleção de espectros e pré-tratamentos matemáticos disponíveis no software Vision ${ }^{\circledR}$

\begin{tabular}{cc}
\hline $\begin{array}{c}\text { Métodos de seleção } \\
\text { de espectros }\end{array}$ & $\begin{array}{c}\text { Pré-tratamentos } \\
\text { matemáticos }\end{array}$ \\
\hline Distância de & $1^{\text {a } \text { derivada }}$ \\
Mahalanobis & $2^{\text {a derivada }}$ \\
Máxima distância & $\begin{array}{c}\text { Eliminação da linha de base } \\
\text { Variável normal }\end{array}$ \\
& padronizada \\
\hline
\end{tabular}

Depois disso, foram desenvolvidos modelos com os pré-tratamentos $1^{\mathrm{a}}$ e $2^{\mathrm{a}}$ derivadas, para se determinar qual delas proporcionaria um melhor modelo. A derivada selecionada foi, então, testada com os outros pré-tratamentos apresentados no Quadro 1, para se analisar se a utilização de mais de um pré-tratamento promovia melhoria nos modelos de calibração.

A próxima etapa foi definir as regiões mais correlacionadas com os movimentos vibracionais dos aminoácidos presentes no CMP. Para isso, foram desenvolvidos modelos utilizando faixas de 50nm de intervalo, desde a faixa 1100$1150 \mathrm{~nm}$ até $2450-2500 \mathrm{~nm}$, usando o método PLS, juntamente com o método de seleção de espectros e o(s) pré-tratamento(s) definidos nas etapas anteriores.

Os resultados desses modelos desenvolvidos com intervalos de $50 \mathrm{~nm}$ foram associados às regiões observadas na Tab. 1.

Com base no valor de $\mathrm{R}^{2}$ encontrado, as regiões foram classificadas e depois agrupadas, com o intuito de se aumentarem as informações moleculares relacionadas com os compostos de interesse e, assim, serem obtidos melhores modelos de calibração. Para isso, primeiramente foram desenvolvidos modelos utilizando apenas as duas melhores regiões, depois as três melhores, e assim por diante, até o agrupamento de todas as faixas espectrais. Esse procedimento visou determinar se todas as regiões associadas aos compostos de interesse são úteis para a melhoria dos modelos de calibração, ou se alguma(s) delas deveria(m) ser retirada(s) por interferir negativamente nos resultados do modelo.

Das oito marcas utilizadas na etapa de calibração, duas marcas, uma produzida no estado de Goiás e a outra produzida em Minas Gerais, foram empregadas para a validação externa. Contudo, essas marcas foram de lotes fabricados com mais de um mês de diferença dos lotes das respectivas marcas utilizados para a calibração.

As marcas utilizadas na etapa de validação externa foram submetidas às mesmas manipulações realizadas nas amostras do conjunto de calibração. Assim, 48 espectros formaram o conjunto de validação. 
Tabela 1. Regiões e faixas espectrais associadas aos movimentos vibracionais da estrutura dos aminoácidos (Murray e Williams, 1990)

\begin{tabular}{|c|c|c|}
\hline Região & $\begin{array}{c}\text { Faixa espectral } \\
(\mathrm{nm})\end{array}$ & Movimento vibracional associado \\
\hline 1 & $1100-1310$ & $\begin{array}{l}2^{\text {a }} \text { sobretons: absorção desconhecida de vários aminoácidos; } 3^{\text {a }} \\
\text { sobretons: estiramento N-H de todos os aminoácidos; estiramento COO } \\
\text { ou combinação de bandas de vários aminoácidos; }\end{array}$ \\
\hline 2 & $1400-1430$ & $3^{\mathrm{a}}$ sobretons: estiramento $\mathrm{O}-\mathrm{H}$ de grupos $\mathrm{COOH}$ \\
\hline 3 & $1490-1610$ & $\begin{array}{c}1^{\mathrm{a}} \text { sobretons: estiramento } \mathrm{N}-\mathrm{H} \text {; extensão } \mathrm{C}-\mathrm{N} \text { de aminas; } 2^{\mathrm{a}} \text { sobretons: } \\
\text { estiramento } \mathrm{COO}^{-} \text {ou combinação de bandas de aminoácidos ionizados; } \\
\text { estiramento } \mathrm{COO}^{-} \text {ou combinação de bandas de vários aminoácidos; } 3^{\mathrm{a}} \\
\text { sobretons: deformação } \mathrm{N}-\mathrm{H} \text {, aminas primárias e secundárias; } \\
\text { deformação } \mathrm{NH}_{3} \text { de aminoácidos; estiramento } \mathrm{C}-\mathrm{O} \text { do grupo } \mathrm{COOH} \text { de } \\
\text { aminoácidos }\end{array}$ \\
\hline 4 & $1590-1650$ & $1^{\mathrm{a}}$ sobretons: estiramento $\mathrm{N}-\mathrm{H}$ de todos os aminoácidos \\
\hline 5 & $1640-1680$ & $3^{\mathrm{a}}$ sobretons: deformação $\mathrm{NH}_{3}+\mathrm{NH}$ de aminoácidos \\
\hline 6 & $1780-1970$ & $1^{\text {a }}$ sobretons: absorção desconhecida de vários aminoácidos \\
\hline 7 & $2020-2150$ & $\begin{array}{l}2^{\mathrm{a}} \text { sobretons: deformação N-H de aminas primárias e secundárias; } 2^{\mathrm{a}} \\
\text { sobretons: deformação } \mathrm{NH}_{3} \text { de aminoácidos; } 2^{\mathrm{a}} \text { sobretons: estiramento } \\
\text { C-O do grupo } \mathrm{COOH} \text { de aminoácidos }\end{array}$ \\
\hline 8 & $2200-2250$ & $2^{\mathrm{a}}$ sobretons: deformação $\mathrm{NH}_{3}+\mathrm{NH}$ de aminoácidos \\
\hline 9 & $2310-2420$ & $\begin{array}{c}1^{\mathrm{a}} \text { sobretons: estiramento } \mathrm{COO}^{-} \text {ou combinação de bandas de } \\
\text { aminoácidos ionizados; estiramento } \mathrm{COO}^{-} \text {ou combinação de bandas de } \\
\text { vários aminoácidos; } 3^{\mathrm{a}} \text { sobretons: estiramento } \mathrm{C}-\mathrm{N} \text { de aminas primárias; } \\
\text { estiramento } \mathrm{C}-\mathrm{N} \text { de aminas primárias }\end{array}$ \\
\hline
\end{tabular}

As amostras da validação foram determinadas por CLAE, seguindo os procedimentos realizados na calibração. Os valores para o tratamento 0 foram de $178,28 \mathrm{mg} / \mathrm{L}$ e de $237,45 \mathrm{mg} / \mathrm{L}$. Dessa forma, após as realizações das adições de soro de queijo reconstituído aos leites utilizados na validação externa em $1 \%$, $2 \%, 3 \%, 4 \%, 6 \%, 8 \%$ e $10 \%$, foram obtidos valores de CMP entre 178,28 e 491,45mg/L.

As mesmas amostras utilizadas na validação externa foram analisadas pelo método NIRS, por meio do modelo de calibração considerado mais adequado para a determinação do CMP em leite
UAT, por espectroscopia de infravermelho próximo (NIRS).

A capacidade qualitativa do modelo de calibração também foi avaliada. Para isso, foram utilizadas quatro marcas de leite UAT desnatado. De cada uma das quatro marcas foram obtidos dois espectros, totalizando oito espectros.

\section{RESULTADOS E DISCUSSÃO}

Os resultados com os métodos distância de Mahalanobis e máxima distância podem ser visualizados na Tab. 2 .

Tabela 2. Modelos de calibração com distância de Mahalanobis e máxima distância, utilizando-se o método PLS e toda a faixa de 1100 a $2500 \mathrm{~nm}$

\begin{tabular}{ccccc}
\hline Modelo & Método & $\mathrm{R}^{2}$ & PRESS & Fatores \\
\hline 1 & Distância de Mahalanobis & 0,6165 & 1510068,250 & 5 \\
2 & Máxima distância & 0,6073 & 1549479 & 5 \\
\hline
\end{tabular}

O modelo 1, que utilizou a distância de Mahalanobis, apresentou maior valor de $\mathrm{R}^{2} \mathrm{e}$ menor valor de PRESS que o modelo 2, que utilizou a máxima distância. Maior valor de $\mathrm{R}^{2}$ significa maior correlação com os valores da reta obtida, e o menor valor de PRESS significa maior robustez. Assim, o método escolhido deveria ser a distância de Mahalanobis.

Contudo, quando foi empregado o método de Mahalanobis em testes utilizando regiões espectrais menores, o software Vision ${ }^{\circledR}$ apresentou uma mensagem de erro, a qual 
alertava que, pelo número de amostras, deveria ser selecionada uma região com um intervalo maior. A utilização da distância de Mahalanobis só foi permitida quando foram colocadas regiões individuais com pelo menos $500 \mathrm{~nm}$ de intervalo. Já a máxima distância aceitou em seus testes regiões de apenas $2 \mathrm{~nm}$ de intervalo.

Como um dos objetivos foi definir regiões associadas com os movimentos vibracionais dos compostos presentes no CMP, optou-se por usar a máxima distância como método de seleção de amostras. Esse método se mostrou viável por tornar possível oa utilização de faixas com intervalos menores e por apresentar valor de $\mathrm{R}^{2}$ pouco menor $(1,51 \%)$ e valor de PRESS pouco maior $(2,79 \%)$ que os obtidos com a distância de Mahalanobis, o que mostra que modelos desenvolvidos por máxima distância podem ter eficiência semelhante aos obtidos por distância de Mahalanobis.

Em relação ao número de fatores, nos dois modelos foi considerado como adequado o uso de cinco fatores, pois foram associados aos mais baixos valores de PRESS. Se fosse utilizado um número de fatores maior que o recomendado, poderia ocorrer a obtenção de modelos sobreajustados (Ferrão e Davanzo, 2005), e se fosse utilizado um menor número de fatores, poderia ocorrer $\mathrm{o}$ efeito denominado de ajustamento inferior (Carneiro, 2008).
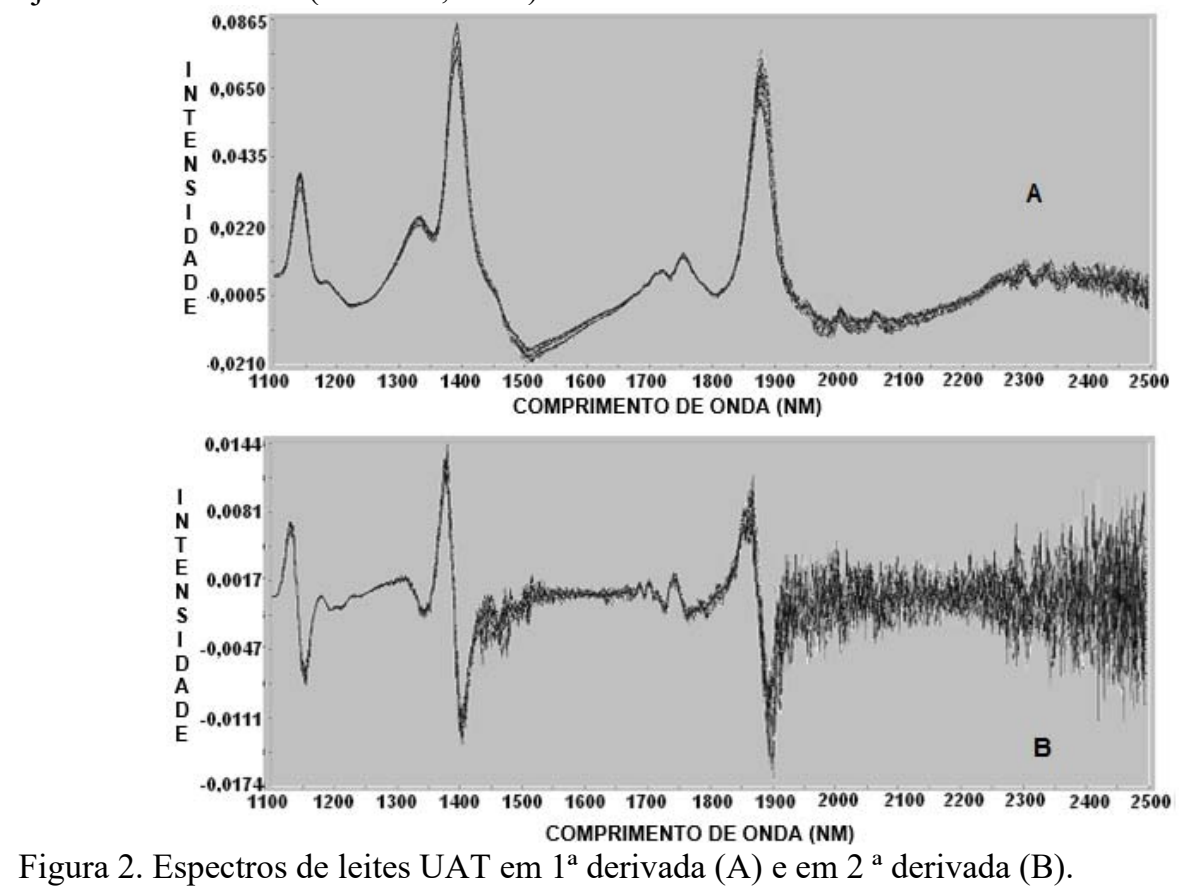

Figura 2. Espectros de leites UAT em $1^{\text {a }}$ derivada (A) e em $2^{\text {a }}$ derivada (B).
O modelo 2, desenvolvido sem a utilização de pré-tratamentos, foi considerado um modelo com baixa capacidade de previsão, já que, de acordo com Moncada (2006), equações de calibração possuem boa capacidade preditiva quando 0 coeficiente de determinação é superior a 0,69. Assim, torna-se imprescindível o uso de prétratamentos de dados, para que os modelos desenvolvidos apresentem maior habilidade de previsão.

Pode-se visualizar na Fig. 2(A) que, nos espectros do conjunto de calibração com a aplicação da $1^{\mathrm{a}}$ derivada, permanece a inclinação da linha de base, apesar de o desvio da linha de base ter sido eliminado. Já nos espectros com a utilização da $2^{\mathrm{a}}$ derivada (Fig. 2-B), observa-se tanto a eliminação do desvio da linha de base, como também da inclinação, obtendo-se espectros que permitem a visualização e interpretação de suas características com maior eficiência. Esses fatores podem justificar o desenvolvimento de um modelo de calibração mais adequado na $2^{\text {a }}$ derivada que o obtido com a $1^{\mathrm{a}}$ derivada, já que foi encontrado, para a $2^{\mathrm{a}}$ derivada, um valor de $\mathrm{R}^{2} 39,31 \%$ superior ao da $1^{\mathrm{a}}$ derivada (Tab. 3). A $2^{\mathrm{a}}$ derivada foi, então, testada com os pré-tratamentos variável normal padronizada, correção da linha de base e eliminação de tendências.

Arq. Bras. Med. Vet. Zootec., v.70, n.3, p.946-956, 2018 
A Tab. 3 mostra que o modelo 5 , com $2^{\mathrm{a}}$ derivada e variável normal padronizada, apresentou valor de $\mathrm{R}^{2}$ superior, além de valor de PRESS inferior aos dos outros modelos com $2^{\mathrm{a}}$ derivada, o que mostra que a combinação dos pré-tratamentos $2^{\mathrm{a}}$ derivada e variável normal padronizada foi a mais adequada para a obtenção de bons modelos.

Contribui também, para a escolha do modelo cinco, o fato de que o $\mathrm{R}^{2}$ obtido foi maior que o considerado pela literatura para boa capacidade preditiva (Moncada, 2006) e por terem sido utilizados apenas três fatores. Além disso, foram reconhecidos como outliers e eliminados apenas $4,16 \%$ dos espectros do conjunto de calibração.

Segundo Pasquini (2003), a exclusão de algumas amostras é frequente e, geralmente, contribui para uma melhoria da qualidade da calibração. Os resultados mostrados na Tab. 3 corroboram essa afirmativa, já que o modelo com a $2^{\mathrm{a}}$ derivada e variável normal padronizada apresentou maior quantidade de outliers e melhores resultados que os demais modelos que utilizaram a $2^{\mathrm{a}}$ derivada.

No entanto, é necessário ser prudente, pois a supressão de uma grande quantidade de amostras outliers resulta na diminuição da representatividade (Pasquini, 2003). Fontaneli e colaboradores (2002) mencionaram que, na rotina e calibração do NIRS, a população-alvo está bem representada quando são eliminadas menos de $10 \%$ de outliers, o que mostra que o número de outliers eliminadas pelo modelo cinco foi adequado.

Tabela 3. Pré-tratamentos utilizados com a máxima distância e método de regressão PLS, utilizando-se toda a faixa de 1100 a $2500 \mathrm{~nm}$

\begin{tabular}{|c|c|c|c|c|c|}
\hline Modelo & Pré-tratamento & $\mathrm{R}^{2}$ & PRESS & Fatores & $\begin{array}{l}\text { Outliers } \mathrm{n}^{\circ} \\
(\%)\end{array}$ \\
\hline 3 & $1^{\mathrm{a}}$ derivada & 0,5703 & 2062621 & 3 & 0 \\
\hline 4 & $2^{\mathrm{a}}$ derivada & 0,7945 & 2012293,5 & 3 & $7(3,64)$ \\
\hline 5 & $\begin{array}{c}2^{\mathrm{a}} \text { derivada com variável } \\
\text { normal padronizada }\end{array}$ & 0,8173 & 1978950 & 3 & $8(4,16)$ \\
\hline 6 & $\begin{array}{c}2^{\mathrm{a}} \text { derivada com correção da } \\
\text { linha de base }\end{array}$ & 0,7637 & 2269413,5 & 3 & $2(1,04)$ \\
\hline 7 & $\begin{array}{l}2^{\mathrm{a}} \text { derivada com eliminação de } \\
\text { tendências }\end{array}$ & 0,7951 & 2009452,625 & 3 & $7(3,64)$ \\
\hline
\end{tabular}

Quando se analisam os resultados da Tab. 4, pode-se observar que faixas espectrais da ordem de 50nm não fornecem bons modelos de calibração, uma vez que todos os valores de $\mathrm{R}^{2}$ foram inferiores a 0,69 . A média de $\mathrm{R}^{2}$ encontrada foi de apenas 0,33 . O valor de PRESS também mostra que modelos com intervalos de $50 \mathrm{~nm}$ possuem baixa capacidade de previsão, pois a maioria dos modelos da Tab. 4 apresentou valor de PRESS maior que o obtido com o modelo 5 (Tab. 3), que utilizou a informação de toda a faixa de 1100 a $2500 \mathrm{~nm}$.

Segundo Bullé (2009), faixas pequenas não oferecem bons modelos, pois é necessário um número mínimo de informações moleculares, por isso os resultados nos intervalos de $50 \mathrm{~nm}$ foram ruins. Contudo, alguns intervalos de 50nm (Tab. 4) mostraram-se mais adequados para a obtenção de modelos de previsão, com $\mathrm{R}^{2}$ acima da média
(0,33), o que indica que a associação dessas faixas espectrais com os movimentos vibracionais específicos da estrutura dos aminoácidos presentes no CMP, de acordo com a Tab. 1, poderia melhorar a qualidade dos modelos de calibração.

Da associação dos movimentos vibracionais dos aminoácidos com os resultados da Tab. 4, mediante a utilização dos critérios descritos em material e métodos, foram selecionadas as seguintes regiões do infravermelho próximo: $1100-1310$; 1400-1430; 1490-1550; 1640-1680; 1780-1970; 2020-2100 e 2310-2350nm. De acordo com os resultados apresentados pelos modelos de cada uma, elas foram classificadas em regiões espectrais mais correlacionadas com os movimentos vibracionais dos aminoácidos do CMP, como demonstrado na Tab. 5. 
Determinação do caseinomacropeptídeo...

Tabela 4. Modelos com faixas de 50nm de intervalo, utilizando máxima distância, $2^{\text {a }}$ derivada e variável normal padronizada, e método de regressão PLS

\begin{tabular}{ccccc}
\hline Modelo & Faixa espectral & $\mathrm{R}^{2}$ & PRESS & Fatores \\
\hline 8 & $1100-1150$ & 0,6061 & 1685377,125 & 4 \\
9 & $1150-1200$ & 0,5354 & 1905676,375 & 3 \\
10 & $1200-1250$ & 0,4905 & 2327253,25 & 5 \\
11 & $1250-1300$ & 0,4631 & 2504410,75 & 6 \\
12 & $1300-1350$ & 0,5144 & 1951239,875 & 4 \\
13 & $1350-1400$ & 0,4097 & 2239908 & 3 \\
14 & $1400-1450$ & 0,4141 & 2701997,25 & 6 \\
15 & $1450-1500$ & 0,2927 & 3102543,75 & 3 \\
16 & $1500-1550$ & 0,422 & 2971597,25 & 5 \\
17 & $1550-1600$ & 0,2696 & 3469525,75 & 6 \\
18 & $1600-1650$ & 0,1019 & 3490163,75 & 1 \\
19 & $1650-1700$ & 0,3977 & 2858971,75 & 6 \\
20 & $1700-1750$ & 0,28 & 3102543,75 & 6 \\
21 & $1750-1800$ & 0,3274 & 3151170,75 & 4 \\
22 & $1800-1850$ & 0,378 & 2751492,75 & 4 \\
23 & $1850-1900$ & 0,5574 & 1830194,375 & 4 \\
24 & $1900-1950$ & 0,3598 & 3098328,25 & 5 \\
25 & $1950-2000$ & 0,4021 & 2656292,75 & 4 \\
26 & $2000-2050$ & 0,3383 & 3078423,25 & 6 \\
27 & $2050-2100$ & 0,3374 & 2992234,25 & 6 \\
28 & $2100-2150$ & 0,0742 & 3976009,75 & 1 \\
29 & $2150-2200$ & 0,1088 & 4055214,5 & 1 \\
30 & $2200-2250$ & 0,0931 & 3684292,25 & 1 \\
31 & $2250-2300$ & 0,076 & 3765374,75 & 1 \\
32 & $2300-2350$ & 0,461 & 2661341 & 6 \\
33 & $2350-2400$ & 0,23 & 3434361,5 & 6 \\
34 & $2400-2450$ & 0,0706 & 4207574 & 1 \\
35 & $2450-2500$ & 0,2303 & 3625222,25 & 6 \\
\hline
\end{tabular}

Tabela 5. Classificação das regiões espectrais mais correlacionadas com os movimentos vibracionais dos aminoácidos do CMP

\begin{tabular}{cccccc} 
Modelo & Classificação & Região espectral & $\mathrm{R}^{2}$ & PRESS & Fatores \\
\hline 36 & $1^{\mathrm{a}}$ & $1100-1310$ & 0,6516 & 1739980,25 & 5 \\
37 & $2^{\mathrm{a}}$ & $1780-1970$ & 0,6237 & 2125471,75 & 6 \\
38 & $3^{\mathrm{a}}$ & $1490-1550$ & 0,4671 & 2794317,5 & 6 \\
39 & $4^{\mathrm{a}}$ & $2310-2350$ & 0,4222 & 2658279,75 & 6 \\
40 & $5^{\mathrm{a}}$ & $2020-2100$ & 0,3824 & 3479440,5 & 4 \\
41 & $6^{\mathrm{a}}$ & $1400-1430$ & 0,2177 & 3174990,5 & 2 \\
42 & $7^{\mathrm{a}}$ & $1640-1680$ & 0,12 & 3266338 & 1 \\
\hline
\end{tabular}

Como não foram obtidos valores de $\mathrm{R}^{2}$ muito próximos, não houve dificuldades para se estabelecerem as melhores regiões, não sendo necessária a análise dos valores de PRESS. Conforme a classificação obtida, essas regiões espectrais foram agrupadas, apresentando modelos com os resultados mostrados na Tab. 6 .
Os resultados da Tab. 6 demonstram que houve melhoria nos modelos à medida que foi sendo agrupado um número maior de regiões, o que mostra que toda a informação correlacionada com os movimentos vibracionais da estrutura dos aminoácidos é determinante para a obtenção do modelo com maior capacidade de previsão. 
Oliveira et al.

Tabela 6. Resultados dos agrupamentos realizados conforme a classificação das melhores regiões espectrais, mostrada na Tab. 5

\begin{tabular}{cccccc} 
Modelo & $\begin{array}{c}\text { Número de regiões } \\
\text { agrupadas }\end{array}$ & $\mathrm{R}^{2}$ & PRESS & Fatores & $\begin{array}{c}\text { Outliers } \\
\left(\mathrm{n}^{\circ} / \%\right)\end{array}$ \\
\hline 43 & Duas & 0,707 & 1857104,125 & 6 & $1(0,52)$ \\
44 & Três & 0,7416 & 1760862,875 & 6 & $2(1,04)$ \\
45 & Quatro & 0,8381 & 1631780,25 & 6 & $2(1,04)$ \\
46 & Cinco & 0,8417 & 1631219 & 5 & $2(1,04)$ \\
47 & Seis & 0,8876 & 1559730,875 & 6 & $2(1,04)$ \\
48 & Sete & 0,8946 & 1323569,25 & 6 & $3(1,56)$ \\
\hline
\end{tabular}

Assim, o modelo 48, desenvolvido com o método de seleção máxima distância, com os pré-tratamentos $2^{\mathrm{a}}$ derivada e variável normal padronizada, com o método de regressão PLS, utilizando as regiões espectrais 1100-1310; $1400-1430$; 1490-1550; 1640-1680; 1780-1970; 2020-2100 e 2310-1350nm, foi considerado o mais adequado para a determinação de CMP em leite UAT, pois, entre todos os modelos desenvolvidos, foi o que apresentou o maior valor de $\mathrm{R}^{2}$ e o menor valor de PRESS.
A relação entre os valores determinados por esse modelo e os valores obtidos pela análise de referência (CLAE) é mostrada na Fig. 3. Esses resultados mostram o ajuste dos dados obtidos pelo método de calibração, com os dados do método de referência, sendo o ajuste equivalente ao $\mathrm{R}^{2}$ de 0,8946 , o maior valor alcançado nos modelos testados.

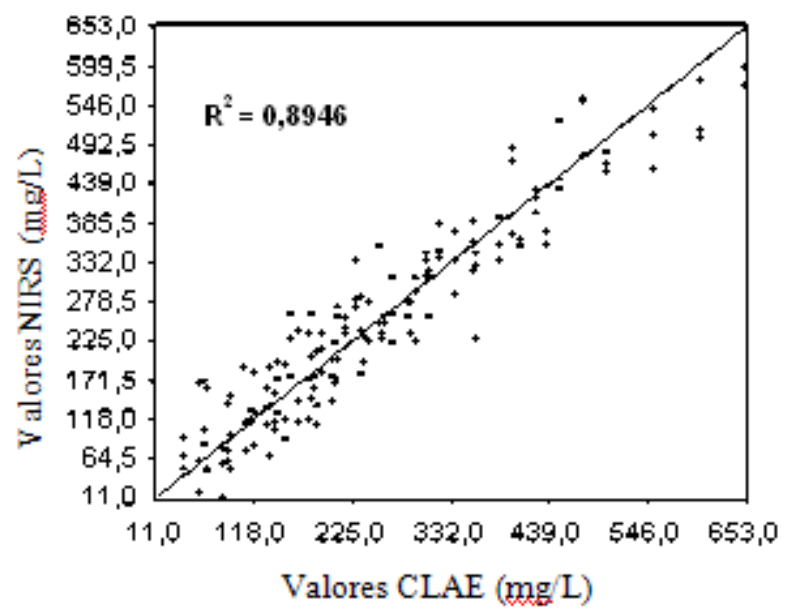

Figura 3. Relação entre os valores determinados pelo NIRS e os valores de referência obtidos por CLAE.

Os 48 espectros do conjunto de validação foram reconhecidos pelo NIRS. O valor do erro-padrão de calibração (SEC) do modelo utilizado (modelo 48) foi de 98,3677. O erro-padrão de validação (SEV) foi de 132,472. Assim, a razão $\mathrm{SEC} / \mathrm{SEV}$ foi de 0,74 , o que evidencia que o modelo de calibração foi bem ajustado.

Nenhum dos espectros das marcas de leite UAT desnatado, utilizados para avaliar a capacidade qualitativa do modelo de calibração, foi reconhecido pelo modelo NIRS, o que mostra que o modelo apresentou boa capacidade de distinção.

Então, provavelmente, o banco de dados de leites UAT não servirá para a determinação de CMP em outro tipo de leite fluido, já que a composição desses leites é influenciada por especificidades do processamento.

Mesmo em leite UAT, há necessidade de se obter um conjunto de calibração mais amplo, com amostras representativas de toda a população. Ainda que haja variação entre as oito marcas 
utilizadas para a calibração, só foram utilizadas amostras de um único lote e de um mesmo período de produção. Como ocorrem diferenças na composição do leite, por influência das estações, da temperatura, das condições climáticas, da alimentação, etc., é possível que seja necessária a utilização de grande quantidade de amostras, de diferentes épocas do ano, a fim de se obter variabilidade suficiente para representar toda a faixa de trabalho.

Laboratórios ou indústrias que empregam na rotina o método CLAE para a determinação de CMP em leite podem utilizar as amostras recebidas para a formação do conjunto de calibração por NIRS. Geralmente, esses laboratórios processam grande quantidade de amostras, com variação suficiente para representar toda a população de estudo. Além disso, a utilização do método de referência em todas as amostras vai garantir resultados mais exatos e precisos.

\section{CONCLUSÕES}

O método de seleção de espectros máxima distância e os pré-tratamentos $2^{\mathrm{a}}$ derivada e variável normal padronizada, juntamente com o método de regressão PLS, são ferramentas quimiométricas eficientes para a obtenção de bons modelos de calibração para a determinação de CMP em leite UAT. Nem todas as regiões do infravermelho próximo apresentaram-se adequadas, sendo necessária a seleção das regiões espectrais mais correlacionadas com os movimentos vibracionais específicos da estrutura dos aminoácidos do CMP, em leite UAT. A espectroscopia de infravermelho próximo pode ser uma alternativa para a determinação de CMP em leite UAT, desde que haja um conjunto de calibração com amostras representativas da população a ser predita no futuro.

\section{AGRADECIMENTOS}

Ao Centro de Pesquisa em Alimentos, da Escola de Veterinária e Zootecnia da Universidade Federal de Goiás, que, com recursos próprios, possibilitou a execução das análises laboratoriais realizadas neste estudo.

\section{REFERÊNCIAS}

BOTELHO, B.G.; REIS, N.; OLIVEIRA, L.S.; SENA, M.M. Development and analytical validation of a screening method for simultaneous detection of five adulterants in raw milk using mid-infrared spectroscopy and PLSDA. Food Chem., v.181, p.31-37, 2015.

BRASIL. Determinação de Índice de CMP por SEC. 2014. Disponível em: $<$ http://www.agricultura.gov.br/arq_editor/file/A niamal/Laborat\%C3\%B3rios/Metodos\%20IQA/P OA/Leite $\% 20 \mathrm{e} \% 20$ Produtos $\% 20$ Lacteos/MET\% 20POA\%2004\%2003\%20CMP\%20POA.pdf>. Acessado em: 27 dez. 2016.

BRASIL. Ministério da Agricultura, Pecuária e Abastecimento. Instrução Normativa $\mathrm{n}^{\circ}$ 69, 13 dez. 2006a. Institui critério de avaliação da qualidade do leite in natura, concentrado e em pó, reconstituídos, com base no método analítico oficial físico-químico denominado "Índice CMP”. Disponível em: $<$ http://extranet.agricultura.gov.br/sislegisconsulta/consultarLegislacao.do?>. Acessado em: 03 set. 2009.

BRASIL. Ministério da Agricultura, Pecuária e Abastecimento. Instrução Normativa $n^{0}$ 68, 12 dez. 2006b. Oficializa os métodos analíticos oficiais físico-químicos, para controle de leite e produtos lácteos. Disponível em: $<$ http://extranet.agricultura.gov.br/sislegisconsulta/consultarLegislacao.do?>. Acessado em: 03 set. 2009.

BULLÉ, D.J. Tecnologias limpas na síntese $e$ avaliação toxicológica de 1,2,4-oxadiazóis em Aspergillus niger. 2009. 110f. Dissertação (Mestrado em Tecnologia Ambiental) Universidade de Santa Cruz do Sul, Santa Cruz do Sul, RS.

CARNEIRO, M.E. Classificação de lâminas de madeira de pinus spp por espectroscopia óptica. 2008. 97f. Dissertação (Mestrado em Engenharia Florestal) - Universidade Federal do Paraná, Curitiba, PR.

CARVALHO, B.M.A.; CARVALHO, L.M.; ALCÂNTRA, L.A.P.; BONOMO, R.C.F. Métodos de detecção de fraude em leite por adição de soro de queijo. Ver. Eléctron. Vet., v.8, n.6, 7p., 2007. 
CIPOLAT-GOTET, C.; CECCHINATO, A.; DE MARCHI, M. et al. Comparison between mechanical and near-infrared methods for assessing coagulation properties of bovine milk. J. Dairy Sci., v.95, p.6806-6819, 2012.

FERRÃO, M.F.; DAVANZO, C.U. Horizontal attenuated total reflection applied to simultaneous determination of ash and protein contents in commercial wheat flour. Anal. Chim. Acta, v.440, p.411-415, 2005.

FERRÃO, M.F.; MELLO, C.; BORIN, A. et al. LS-SVM: uma nova ferramenta quimiométrica para regressão multivariada. Comparação de modelos de regressão LS-SVM e PLS na quantificação de adulterantes em leite em pó empregando NIR. Quim. Nova, v.30, p.852-859, 2007.

FONTANELI, R.S.; DURR, J.W.; SCHEFFERBASSO, S.M. et al. Validação do método da reflectância no infravermelho proximal para análise de silagem de milho. Rev. Bras. Zootec., v.31, p.594-598, 2002.

KLANDAR, A.H.; LAGAUDE, A.; CHEVALIER-LUCIA, D. Assessment of the rennet coagulation of skim milk: a comparison of methods. Int. Dairy J., v.17, p.1151-1160, 2007.

LI, L.; DING, W. Discriminant analysis of raw milk adulterated with botanical filling material using near infrared spectroscopy. Guang Pu Xue Yu Guang Pu Fen Xi, v.30, p.1238-1242, 2010.

LYNDGAARD, C.B.; ENGELSEN, S.B.; VAN DEN BERG, F.W.J. Real-time modeling of milk coagulation using in-line near infrared spectroscopy. J. Food Eng., v.108, p.345-352, 2012.
MONCADA, G.V.H. Espectroscopía de reflectancia en el infrarrojo cercano (NIRS) en el análisis cuantitativo y cualitativo de carne de cordero. 2006. 126f. Dissertação (Mestrado em Produção Animal) - Universidade Austral de Chile, Valdivia, CHI.

NATALINO, R. Caracterização de açúcar mascavo aplicando análise das componentes principais a dados espectrométricos. 2006. 52f. Dissertação (Mestrado em Agroquímica) Universidade Federal de Viçosa, Viçosa, MG.

PASQUINI, C. Near infrared spectroscopy: Fundamentals practical aspects and analytical applications. J. Braz. Chem. Soc., v.14, p.198219, 2003.

SKOOG. D.A.; HOLLER, F.J.; NIEMAN, T.A. Princípios de análise instrumental. 5.ed. São Paulo: Oficial da Sociedade Brasileira de Química, 2002.836p.

VALENTE， G.F.S.; GUIMARÃES， D.C.; GASPARDI, A.L.A.; OLIVEIRA, L.A. Aplicação de redes neurais artificiais como teste de detecção de fraude de leite por adição de soro de queijo. Rev. Inst. Laticínios Cândido Tostes, v.69, p.425-432, 2014.

VAN KEMPEN, T.A.T.G.; JACKSON, D. NIRS may provide rapid evaluation of amino acids. Feedstuffs, v.68, p.12-15, 1996.

VARMUZA, K.; FILZMOSER, P. Introduction to multivariate statistical analysis in chemometrics. Boca Raton: CRC Press, 2009.

ZHANG, L.G.; ZHANG, X.; NI, L.J. et al. Rapid identification of adulterated cow milk by nonlinear pattern recognition methods based on near infrared spectroscopy. Food Chem., v.145, p.342-348, 2014. 